\title{
GINS2 regulates the proliferation and apoptosis of colon cancer cells through PTP4A1
}

\author{
$\mathrm{HAO} \mathrm{HU}^{1 *}$, LINA YE ${ }^{*}$ and ZHE LIU ${ }^{2}$ \\ ${ }^{1}$ Department of Endoscopy, Guizhou Provincial People's Hospital, Guiyang, Guizhou 550002; \\ ${ }^{2}$ Department of Gastroenterology, Affiliated Cancer Hospital of Guizhou Medical University, \\ Guiyang, Guizhou 550001, P.R. China
}

Received November 16, 2021; Accepted December 24, 2021

DOI: $10.3892 / \mathrm{mmr} .2022 .12633$

\begin{abstract}
Colon cancer is associated with high death rates worldwide and poses a serious threat to public health. GINS complex subunit 2 (GINS2) serves a carcinogenic role in many cancers, including gastric adenocarcinoma, ovarian cancer and pancreatic cancer. However, the specific function of GINS2 in the development of colon cancer has not been described in detail. The present study aimed to clarify the role of GINS2 in colon cancer. A Cell Counting Kit-8 assay, EdU staining, TUNEL and flow cytometry analyses were performed to determine the levels of cell viability, proliferation and apoptosis and to evaluate the cell cycle. Through the analysis of BioGrid, a Protein-Protein Interaction database, it was hypothesized that protein tyrosine phosphatase 4A1 (PTP4A1) is a protein that might interact with GINS2, which was then validated using a co-immunoprecipitation assay. mRNA and protein levels were measured using reverse transcription-quantitative PCR and western blotting, respectively. The results of the present study demonstrated that GINS2 expression levels were increased in colon cancer cells. Furthermore, GINS2 knockdown inhibited the proliferation of colon cancer cells, while the levels of cell cycle arrest and apoptosis were increased. By interacting with PTP4A1, GINS2 promoted the expression of PTP4A1, a novel p53 target. GINS2 knockdown was increased, while PTP4A1 overexpression decreased the protein level of p53. Notably, PTP4A1 overexpression partly reversed the effects of GINS2 downregulation on colon cancer cells. Therefore, the present study demonstrated that GINS2 regulated the proliferation and apoptosis of colon cancer cells through PTP4A1/p53 pathway,
\end{abstract}

Correspondence to: Dr Zhe Liu, Department of Gastroenterology, Affiliated Cancer Hospital of Guizhou Medical University, 1 West Beijing Road, Guiyang, Guizhou 550001, P.R. China E-mail: zheLiu123@126.com

*Contributed equally

Key words: GINS complex subunit 2, protein tyrosine phosphatase 4A1, colon cancer, apoptosis highlighting that GINS2 may serve as a novel molecular marker for colon cancer prevention and therapy.

\section{Introduction}

Colon cancer is a common, highly malignant type of cancer characterized by a high morbidity rate and a poor prognosis (1). According to statistics obtained from the American Cancer Society, human colon cancer has an incidence rate of $10.2 \%$ and a mortality rate of $9.2 \%$ worldwide, ranking it as the fourth most common cancer $(2,3)$. Although advances have been made in the current treatment options available for colon cancer, the associated mortality rates continue to increase and the current 5-year survival rate remains low $(4,5)$. Risk factors associated with colon cancer include age and diet; however, the overexpression of oncogenes and the inactivation of tumor suppressor genes remain the most important contributors (6). At present, conventional therapies, such as surgery, chemotherapy and antibody therapies have been adopted to protect against colon cancer (7). However, these methods exhibit low levels of effectiveness in clinical practice (8). Thus, the present study aimed to investigate the mechanisms underlying the development and progression of colon cancer, which may help to determine effective therapeutic options.

GINS complex subunit 2 (GINS2), also known as PSF2, belongs to the GINS family and acts as a vital participant in both DNA duplication and cell cycle progression $(9,10)$. In addition, GINS2 has been found to serve a key role in the tumorigenesis of various cancers (11). For example, GINS2 is overexpressed in breast cancer cell lines and GINS2 knockdown inhibited breast cancer growth and metastasis (12). In addition, Yan et al (13) demonstrate that GINS2-regulated cell proliferation and apoptosis in human epithelial ovarian cancer. GINS2 is highly expressed in colon cancer $(14,15)$, but the specific role is yet to be fully elucidated.

The results of previous studies demonstrated that phosphatase of regenerating liver 1 (PTP4A1) is upregulated in numerous tumor cells and is involved in promoting both cell migration and invasion (16-18). The analysis of BioGrid (https://thebiogrid.org/), a protein-protein interaction database, suggested that PTP4A1 could interact with GINS2. Results from a previous study demonstrate that PTP4A1 is highly expressed in colon cancer, but not in normal colon tissues or 
colonic adenomas (19). In addition, GINS2 inhibition regulates the proliferation and apoptosis of non-small cell lung cancer cells through p53 (20). PTP4A1 is considered as a novel p53 target and PTP4A1 inhibition increases p53 expression level (21).

Therefore, the present study aimed to explore the role of GINS2 in the proliferation and apoptosis of colon cancer and its potential regulatory mechanism on the PTP4A1/p53 pathway.

\section{Materials and methods}

Cell culture, treatment and transfection. Normal human intestinal epithelial cell line (HIEC-6) and colon cancer cell lines (HCT116, LS174T, HCT8 and SW620) were purchased from the American Type Culture Collection. Cells were incubated in DMEM supplemented with 10\% FBS (both from Gibco; Thermo Fisher Scientific, Inc.), $100 \mathrm{U} / \mathrm{ml}$ penicillin and $100 \mu \mathrm{g} / \mathrm{ml}$ streptomycin (Invitrogen; Thermo Fisher Scientific, Inc.) at $37^{\circ} \mathrm{C}$ in a humidified atmosphere with $5 \% \mathrm{CO}_{2}$.

To knockdown GINS2, short hairpin RNA (shRNA)-GINS2-1/2 and its negative control (shRNA-NC) were designed and synthesized by Shanghai GenePharma Co., Ltd. and shRNA fragments were cloned into a lentiviral GV493 vector (Shanghai GeneChem Co., Ltd.). The target sequences were as follows: shRNA-GINS2-1, 5'-GATTAA CCTGAAACA AAGA-3'; shRNA-GINS2-2, 5'-ATCAAC ACCAGCGGGACTTTC-3'; shRNA-NC, 5'-TTTCTCCGA ACGTGTCACGT-3'. To overexpression PTP4A1, the PTP4A1 coding sequence was synthesized and cloned into a lentiviral GV492 vector. The empty GV492 vector was considered as a negative control (Ov-NC). When 293T cells were cultured to 60-70\% confluency, according to the manufacturer's protocol, a total of $1 \mu \mathrm{g}$ lentiviral plasmid, $1 \mu \mathrm{g}$ 3rd generation viral packaging vectors and $5 \mu 1$ Lipofectamine ${ }^{\circledR} 2000$ transfection reagent (Invitrogen; Thermo Fisher Scientific, Inc.) in serum-free DMEM was incubated for $15 \mathrm{~min}$ at room temperature and then added into 293T cells to amplify at $37^{\circ} \mathrm{C}$ for $6 \mathrm{~h}$. After transfection for $48 \mathrm{~h}$, the supernatant containing virus was collected by centrifugation at $10,000 \mathrm{xg}$ for $4 \mathrm{~h}$ at $4^{\circ} \mathrm{C}$. HCT116 cells at the 3 rd passage were infected with lentiviral vectors at an MOI of 50 in the presence of $5 \mu \mathrm{g} / \mathrm{ml}$ polybrene (MilliporeSigma) for $24 \mathrm{~h}$ at $37^{\circ} \mathrm{C}$. Subsequently, the medium was replaced with fresh medium and the stable cells were selected with $2 \mu \mathrm{g} / \mathrm{ml}$ puromycin for 3 days. The transfection efficiency was evaluated by reverse transcription-quantitative (RT-q) PCR and western blotting.

$R T-q P C R$. Total RNA was extracted from $1 \times 10^{6}$ cells using TRIzol ${ }^{\circledR}$ reagent (Invitrogen; Thermo Fisher Scientific, Inc.) and subsequently reverse transcribed into cDNA using the PrimeScript RT Reagent kit (Takara Biotechnology Co., Ltd.) according to the manufacturer's protocols under the following conditions: $37^{\circ} \mathrm{C}$ for $15 \mathrm{~min}$ and at $85^{\circ} \mathrm{C}$ for $5 \mathrm{sec}$. The synthesized cDNA was used as a template for PCR, which was performed using an ABI 7000 quantitative PCR instrument (Applied Biosystems; Thermo Fisher Scientific, Inc.) using the SYBR Green PCR kit (Applied Biosystems; Thermo Fisher Scientific, Inc.). The following thermocycling conditions were used for the qPCR: Initial denaturation at $95^{\circ} \mathrm{C}$ for $6 \mathrm{~min}$; followed by 40 cycles of denaturation at $95^{\circ} \mathrm{C}$ for $15 \mathrm{sec}$, annealing at $60^{\circ} \mathrm{C}$ for $30 \mathrm{sec}$ and extension at $72^{\circ} \mathrm{C}$ for $45 \mathrm{sec}$ and a final extension at $72^{\circ} \mathrm{C}$ for $5 \mathrm{~min}$. The sequences of the gene primers were as follows: GINS2 forward, 5'-CAGAAA TGTCGCCTGCTCC-3' and reverse, 5'-GGATTTCGTCTG CCTTCG-3'; PTP4A1 forward, 5'-ATTGAAGGTGGAATG AAATACGAAG-3' and reverse, 5'-TACTTCTCCAAATAC AGAAGTTGCT-3'; and GAPDH forward, 5'-ACCTGACCT GCCGTCTAGAAAA-3' and reverse, 5'-TTGAAGTCAGAG GAGACCACCTG-3'. Relative mRNA expression levels were quantified using the $2^{-\Delta \Delta C q}$ method (22) and normalized to the internal reference gene GAPDH.

Western blotting. Total protein was extracted from the cells using RIPA lysis buffer (Beyotime Institute of Biotechnology) and subsequently quantified using BCA kits (Thermo Fisher Scientific, Inc.). Proteins (30 $\mu \mathrm{g} / \mathrm{lane})$ were separated on a $10 \%$ gel by SDS-PAGE and subsequently transferred onto PVDF membranes. The membranes were blocked with $5 \%$ non-fat milk for $1 \mathrm{~h}$ at room temperature and the proteins were incubated with primary antibodies against GINS2 (1:500; cat. no. ab197123; Abcam), PTP4A1 (1:400; cat. no. ab121185; Abcam), p21 (1:1,200; cat. no. ab109520; Abcam), cyclin D1 (1:200; cat. no. ab16663; Abcam), Bcl2 (1:1,500; cat. no. ab182858; Abcam), Bax (1:1,500; cat. no. ab182733; Abcam), poly (ADP-ribose) polymerase (PARP) (1:1,000; cat. no. ab191217; Abcam), p53 (1:5,000; cat. no. ab32389; Abcam $)$ and GAPDH $(1: 2,500$; cat. no. ab 9485 ; Abcam $)$ at $4^{\circ} \mathrm{C}$ overnight. Following primary incubation, the membranes were incubated with an HRP-conjugated goat anti-rabbit secondary antibody (1:20,000; cat. no. ab205718; Abcam) for $2 \mathrm{~h}$ at room temperature. Protein bands were visualized using enhanced chemiluminescence (MilliporeSigma) and imaging system (Tanon-5200; Tanon Science and Technology Co., Ltd.). The gray values of the bands were semi-quantified using ImageJ software (version 1.0; National Institutes of Health) and the protein expression was normalized against GAPDH.

Cell Counting Kit-8 (CCK-8). Transfected cells $\left(5 \times 10^{3}\right.$ cells/well) were inoculated into 96 -well plates. CCK-8 reagent (10 $\mu \mathrm{l} /$ well; Beyotime Institute of Biotechnology) was added and the cells were incubated at $37^{\circ} \mathrm{C}$ for $2 \mathrm{~h}$. Subsequently, cell viability at 24,48 or $72 \mathrm{~h}$ was evaluated using a microplate reader at $450 \mathrm{~nm}$.

5-ethynyl-2'-deoxyuridine (EdU) staining. Transfected cells $\left(5 \times 10^{4}\right.$ cells/well) were inoculated into $96-$ well plates and incubated with $20 \mu \mathrm{M}$ EdU (Thermo Fisher Scientific, Inc.) at $37^{\circ} \mathrm{C}$ for $2 \mathrm{~h}$. Subsequently, cells were fixed and permeated using $4 \%$ paraformaldehyde for $30 \mathrm{~min}$ and $0.5 \%$ Triton X-100 for $10 \mathrm{~min}$ at room temperature, respectively. The cells were stained with Cell-Light ${ }^{\mathrm{TM}}$ EdU Apollo ${ }^{\circledR} 488$ In Vitro Imaging kit (Thermo Fisher Scientific, Inc.) according to the manufacturer's protocol. Cells were observed using a fluorescence microscope (magnification, x200; Nikon Corporation).

Flow cytometry. The harvested cells were fixed overnight with $75 \%$ ethanol at $4^{\circ} \mathrm{C}$ and subsequently cultured with propidium iodide (PI)/RNase staining buffer at $37^{\circ} \mathrm{C}$ for $30 \mathrm{~min}$ in the 

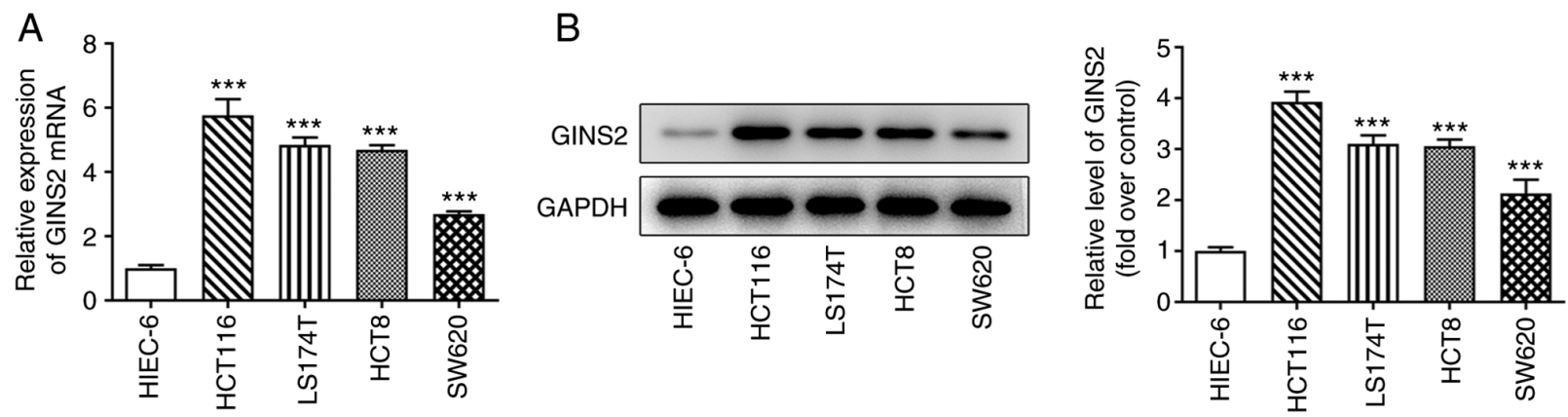

Figure 1. GINS2 is overexpressed in colon cancer cells. (A) GINS2 mRNA expression levels in colon cancer cells were detected using reverse transcription-quantitative PCR. (B) GINS2 protein expression levels in colon cancer cells were detected using western blotting analysis. ${ }^{* * *} \mathrm{P}<0.001$ vs. HIEC-6. GINS2, GINS complex subunit 2 .

dark. The cell cycle analysis was performed using a BD FACSCalibur flow cytometer (BD Biosciences). The distribution of cell cycle, that is, the cell percentages at the $\mathrm{G}_{0} / \mathrm{G}_{1}$, $\mathrm{S}$ and $\mathrm{G}_{2} / \mathrm{M}$ phases, were quantified using FlowJo software (version 7.0; FlowJo LLC).

TUNEL staining. Cell apoptosis was determined using the TUNEL Apoptosis Assay kit (Beyotime Institute of Biotechnology). Briefly, fixation and permeabilization of HCT116 cells were performed using $4 \%$ paraformaldehyde for $20 \mathrm{~min}$ and $0.1 \%$ Triton X-100 for $10 \mathrm{~min}$ at room temperature, respectively. Subsequently, cells were incubated with the TUNEL reaction solution at $37^{\circ} \mathrm{C}$ for $1 \mathrm{~h}$ in the dark and the nuclei were stained with DAPI $(5 \mu \mathrm{g} / \mathrm{ml})$ for $5 \mathrm{~min}$ at room temperature. A total of five visual fields were randomly selected and apoptotic cells were observed on glass coverslips under a fluorescence microscope (magnification, x200; Nikon Corporation).

Co-immunoprecipitation (Co-IP) assay. The interaction between GINS2 and PTP4A1 was determined using a Co-IP assay. Briefly, the harvested cells were fully lysed using $1 \mathrm{ml}$ Co-RIPA buffer (Applygen Technologies, Inc.) and then lysates were pre-cleared with $30 \mu 1$ Protein A/G PLUS-Agarose (Santa Cruz Biotechnology, Inc.) at $4^{\circ} \mathrm{C}$ for $1 \mathrm{~h}$. After centrifuging the lysate, taking the supernatant and removing the beads in the supernatant, immunoprecipitation with antibodies against GINS2 (1:50; cat. no. sc-376595; Santa Cruz Biotechnology, Inc.), PTP4A1 (1:30; cat. no. sc-365659; Santa Cruz Biotechnology, Inc.) and IgG (1:50; cat. no. sc-69786; Santa Cruz Biotechnology, Inc.) was performed with shaking at $4^{\circ} \mathrm{C}$ overnight. The protein A/G-Sepharose beads were added to the samples and shaken at $4^{\circ} \mathrm{C}$ for $4 \mathrm{~h}$. Following centrifugation at $800 \mathrm{x} \mathrm{g}$ for $5 \mathrm{~min}$ at $4^{\circ} \mathrm{C}$, the supernatant was removed and the beads were washed three times $(800 \mu 1 /$ time $)$ with pre-cooled Co-RIPA buffer to obtain protein samples for western blotting analysis.

Statistical analysis. All experiments were performed in triplicate. All data are presented as the mean \pm standard deviation and statistical analysis was performed using SPSS 20.0 (IBM Corp.). Unpaired Student's t-tests were performed to compare the differences between two groups and one-way ANOVA followed by Tukey's post hoc test was used for multiple comparisons. $\mathrm{P}<0.05$ was considered to indicate a statistically significant difference.

\section{Results}

GINS2 is overexpressed in colon cancer cell lines. The expression levels of GINS2 were measured in all colon cancer cells. As depicted in Fig. 1A and B, compared with HIEC-6, the GINS2 mRNA and protein expression levels were increased in colon cancer cell lines and the levels were highest in HCT116 cells. Subsequently, the HCT116 cell line was selected for the following experiments.

GINS2 knockdown inhibits the proliferation of HCT116 cells. HCT116 cells were transfected with shRNA-GINS2 plasmids for GINS2 knockdown and this was subsequently detected using RT-qPCR and western blotting. The results displayed in Fig. 2A and B demonstrated that the mRNA and protein expression levels of GINS2 were significantly reduced in shRNA-GINS2-1 and shRNA-GINS2-2 cells compared with shRNA-NC. GINS2 expression levels were lowest in HCT116 cells transfected with the shRNA-GINS2-1 plasmid. Thus, the shRNA-GINS2-1 plasmid was used for subsequent experiments. The results of the CCK- 8 assay demonstrated that, compared with cells transfected with shRNA-NC, the viability of HCT116 cells was markedly reduced following GINS2 knockdown (Fig. 2C). Similarly, the results of the EdU staining assay demonstrated that transfection with the shRNA-GINS2 plasmid notably inhibited the levels of cell proliferation, compared with HCT116 cells transfected with shRNA-NC (Fig. 2D). In summary, GINS2 promoted colon cancer cell proliferation.

GINS2 knockdown promotes HCT116 cell cycle arrest. The cell cycle was evaluated using flow cytometry. As demonstrated in Fig. 3A, the number of cells in the $\mathrm{G}_{0} / \mathrm{G}_{1}$ phase was increased, while those in $S$ phase and $G_{2}$ phase were decreased following GINS2 knockdown, compared with cells transfected with shRNA-NC. In addition, western blotting was performed to determine the expression levels of cell cycle-associated proteins and the results demonstrated that shRNA-GINS2 reduced the levels of cyclin D1 expression, while p21 expression was increased, compared with cells transfected with 
A

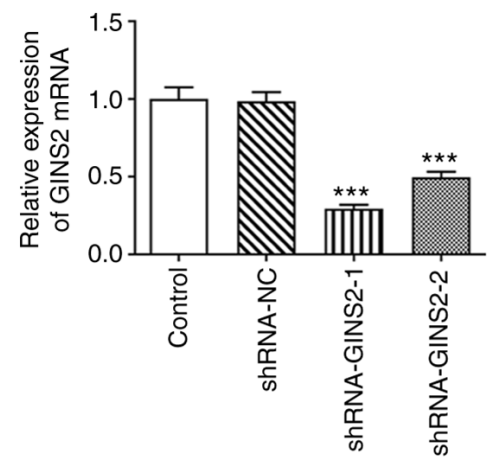

B

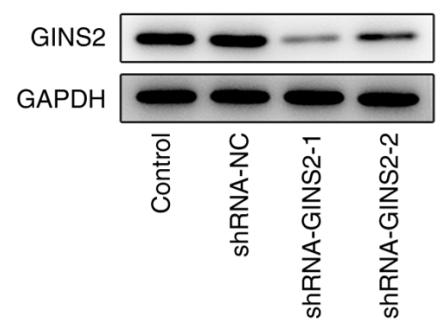

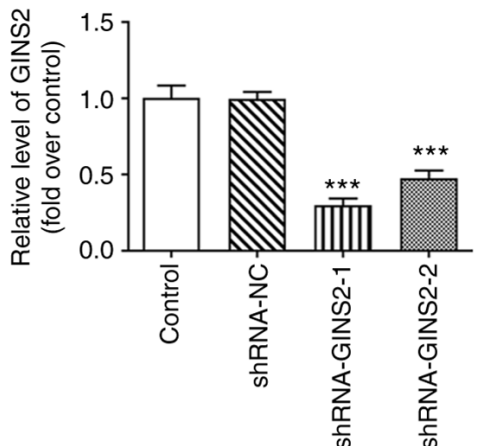
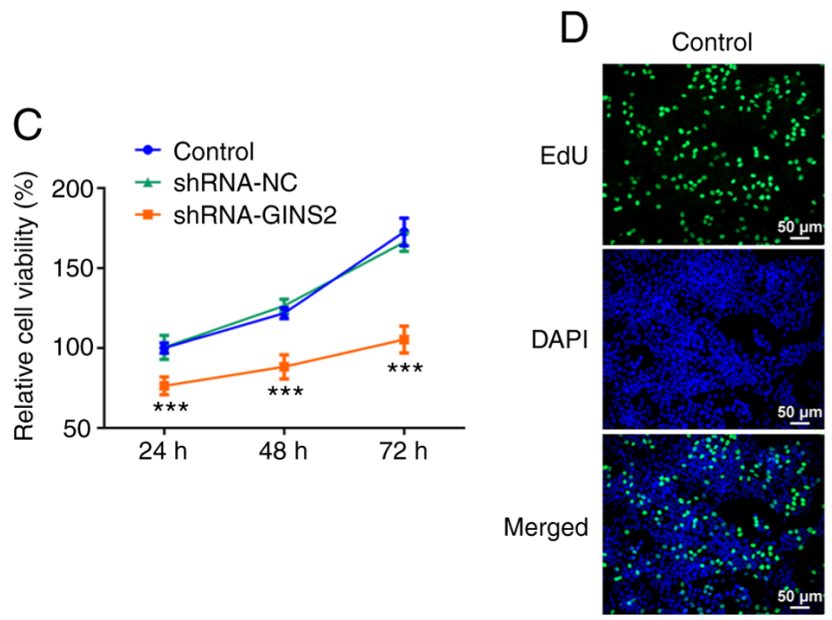

ShRNA-NC ShRNA-GINS2
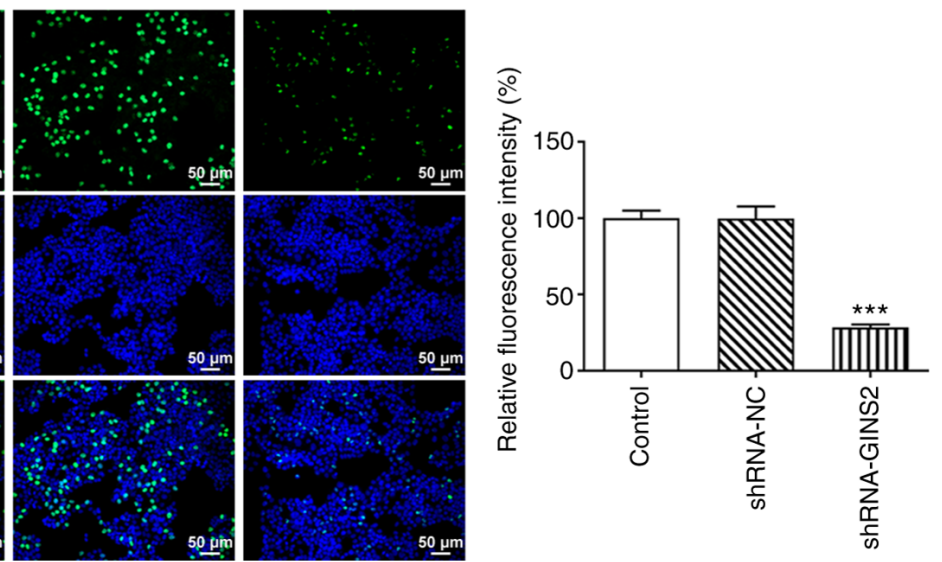

Figure 2. GINS2 knockdown inhibits the proliferation of HCT116 cells. GINS2 (A) mRNA and (B) protein levels in GINS2-knockdown HCT116 cells were detected using reverse transcription-quantitative PCR and western blotting analyses, respectively. (C) Cell Counting Kit- 8 assay was performed to detect the viability of GINS2-knockdown HCT116 cells. (D) EdU staining was performed to detect the proliferation of GINS2-knockdown HCT116 cells. ${ }^{* * *}$ P $<0.001$ vs. shRNA-NC. GINS2, GINS complex subunit 2; shRNA, short hairpin RNA; NC, negative control.

shRNA-NC (Fig. 3B). Collectively, these results demonstrated that GINS2 promoted colon cancer cell cycle progression.

GINS2 knockdown promotes colon cancer cell apoptosis. TUNEL staining was performed to determine the effects of GINS2 on HCT116 cell apoptosis. As demonstrated in Fig. 4A, cell apoptosis was markedly increased following transfection with the shRNA-GINS2 plasmids. In addition, the levels of apoptosis-associated proteins, such as $\mathrm{Bcl} 2$, Bax and cleaved PARP, were measured using western blotting. The results of the present study demonstrated that the expression levels of $\mathrm{Bcl} 2$ were notably reduced following GINS2 knockdown, but the expression levels of Bax and cleaved PARP/PARP were increased, compared with cells transfected with shRNA-NC (Fig. 4B).

GINS2 knockdown activates the p53 pathway through PTP4A1. To determine the expression levels of PTP4A1 in colon cancer cells, RT-qPCR was performed. The results demonstrated that the mRNA expression levels of PTP4A1 were increased in HCT116, LS174T, HCT8 and SW620 cells, compared with the with HIEC-6 cells (Fig. 5A). In addition, a Co-IP assay was performed to verify the targeted binding of GINS2 and PTP4A1. The results of Co-IP demonstrated that GINS2 could interact with PTP4A1 (Fig. 5B). In addition, compared with shRNA-NC, shRNA-GINS2 reduced
PTP4A1 expression level and increased p53 expression level (Fig. 5C).

Following transfection with Ov-PTP4A1 plasmid, the mRNA and protein levels of PTP4A1 were detected using RT-qPCR and western blotting, respectively. Compared with cells transfected with Ov-NC, PTP4A1 expression levels were increased in Ov-PTP4A1 (Fig. 5D). In addition, the results displayed in Fig. 5E demonstrated that shRNA-GINS2 upregulated the expression levels of p53, which were subsequently reversed following transfection with the Ov-PTP4A1 plasmid.

GINS2 knockdown regulates cell proliferation, cycle arrest and apoptosis in colon cancer cells through PTP4A1/p53 pathway. As displayed in Fig. 6A and B, the shRNA-GINS2-mediated decrease in cell proliferation was increased following PTP4A1 overexpression. In addition, shRNA-GINS2 markedly decreased the number of cells in the $G_{0} / G_{1}$ phase and decreased the number of cells in the $S$ phase and $G_{2}$ phase. Notably, this effect was inhibited following PTP4A1 overexpression (Fig. 6C), which highlighted that the cell cycle arrest induced by shRNA-GINS2 was subsequently restored following PTP4A1 overexpression. The results of the TUNEL assay revealed that apoptosis was increased in shRNA-GINS2; however, this effect was inhibited following transfection with Ov-PTP4A1 (Fig. 6D). Furthermore, the western blotting analysis results demonstrated that the shRNA-GINS2-mediated 

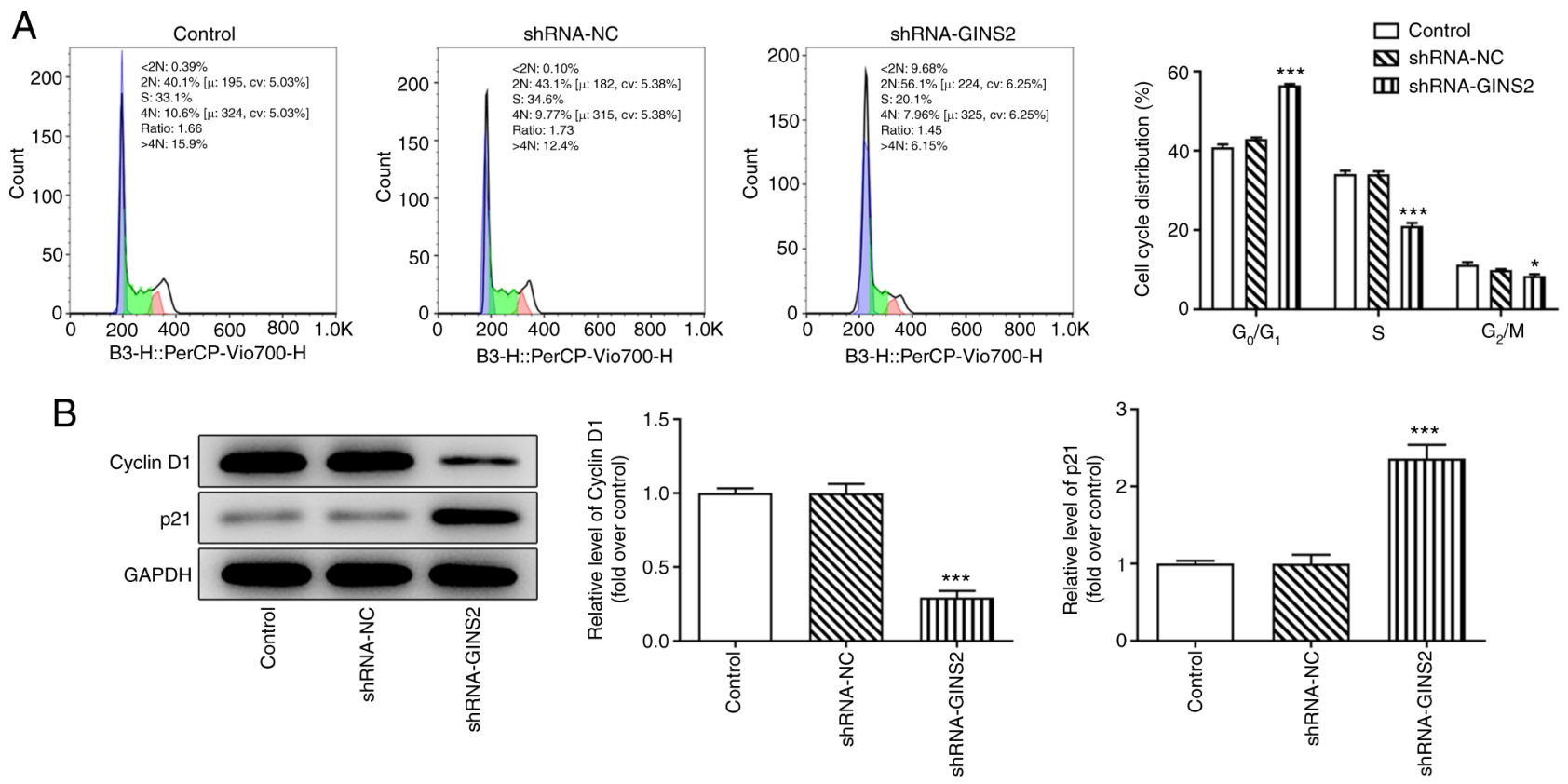

Figure 3. GINS2 knockdown promotes cell cycle arrest in colon cancer. (A) Flow cytometry analysis was performed to examine the cell cycle distribution of GINS2-knockdown HCT116 cells. (B) Western blotting was performed to detect the protein expression levels of p21 and cyclin D1. ${ }^{*} \mathrm{P}<0.05,{ }^{* * *} \mathrm{P}<0.001$ vs. shRNA-NC. GINS2, GINS complex subunit 2; shRNA, short hairpin RNA; NC, negative control.

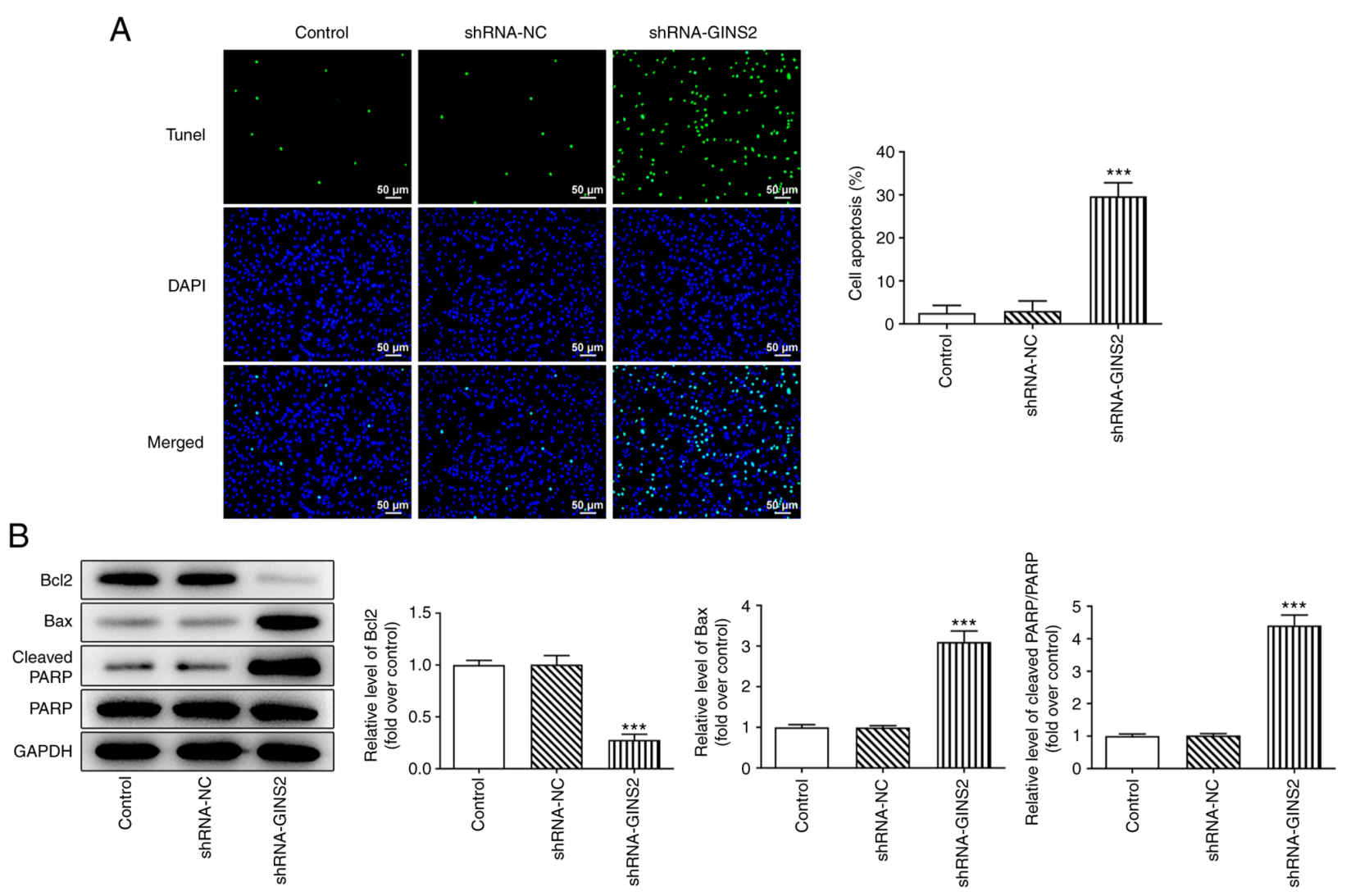

Figure 4. GINS2 knockdown promotes the apoptosis of colon cancer cells. (A) TUNEL staining was performed to detect the apoptosis of GINS2-knockdown HCT116 cells. (B) Western blotting was performed to detect the protein expression levels of Bcl2, Bax, cleaved PARP and PARP. ${ }^{* * *} \mathrm{P}<0.001$ vs. shRNA-NC. GINS2, GINS complex subunit 2; shRNA, short hairpin RNA; NC, negative control; PARP, poly ADP-ribose polymerase.

reduction in the protein expression levels of cyclin D1 and $\mathrm{Bcl} 2$ and the increased protein expression levels of $\mathrm{p} 21$, Bax, cleaved PARP/PARP, were rescued following PTP4A1 overexpression (Fig. 6E and F). These results indicated that GINS2 modulated colon cancer cell proliferation, cycle arrest and apoptosis through the PTP4A1/p53 pathway (Fig. 6G). 

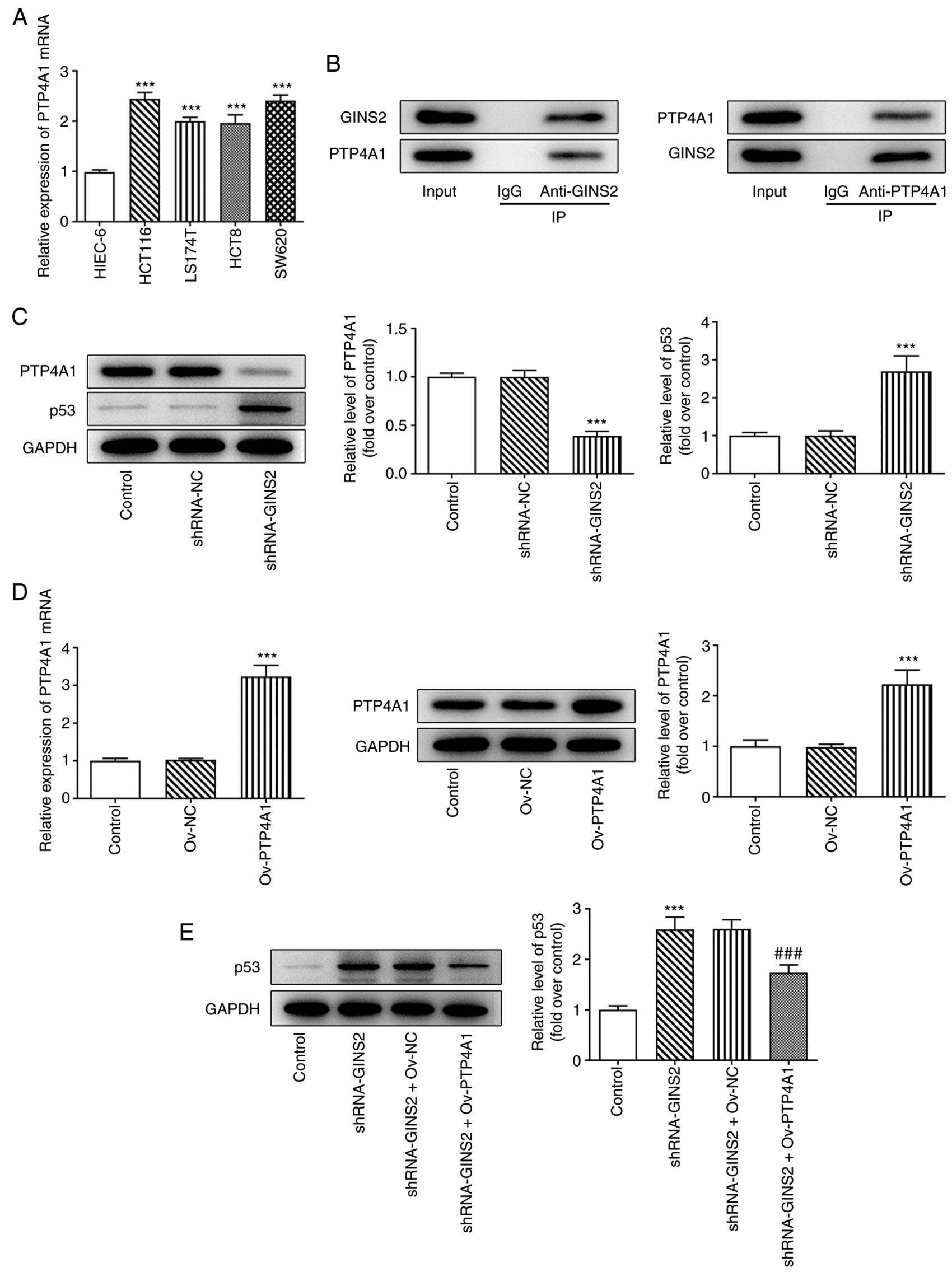

Figure 5. GINS2 knockdown activates the p53 pathway through PTP4A1. (A) RT-qPCR was performed to detect the PTP4A1 mRNA expression level in colon cancer cells. ${ }^{* * *} \mathrm{P}<0.001$ vs. HIEC-6. (B) The binding of GINS2 and PTP4A1 was detected using a co-immunoprecipitation assay. (C) mRNA and protein expression levels of PTP4A1 and p53 were detected using RT-qPCR and western blotting, respectively. ${ }^{* * *} \mathrm{P}<0.001$ vs. shRNA-NC. (D) PTP4A1 mRNA and protein expression levels were detected using RT-qPCR and western blotting, respectively. ${ }^{* * *} \mathrm{P}<0.001$ vs. Ov-NC. (E) p53 protein expression levels were detected using western blotting analysis. ${ }^{* * *} \mathrm{P}<0.001$ vs. Control; ${ }^{\# \# \#} \mathrm{P}<0.001$ vs. shRNA-GINS2 + Ov-NC. GINS2, GINS complex subunit 2; PTP4A1, protein tyrosine phosphatase 4A1; RT-qPCR, reverse transcription-quantitative PCR; shRNA, short hairpin RNA; NC, negative control; Ov, overexpression. 
A

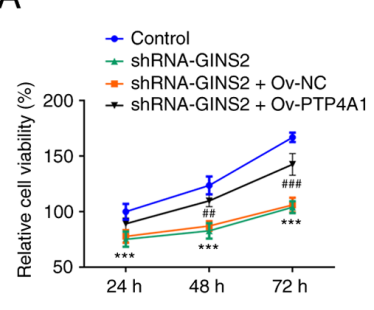

B
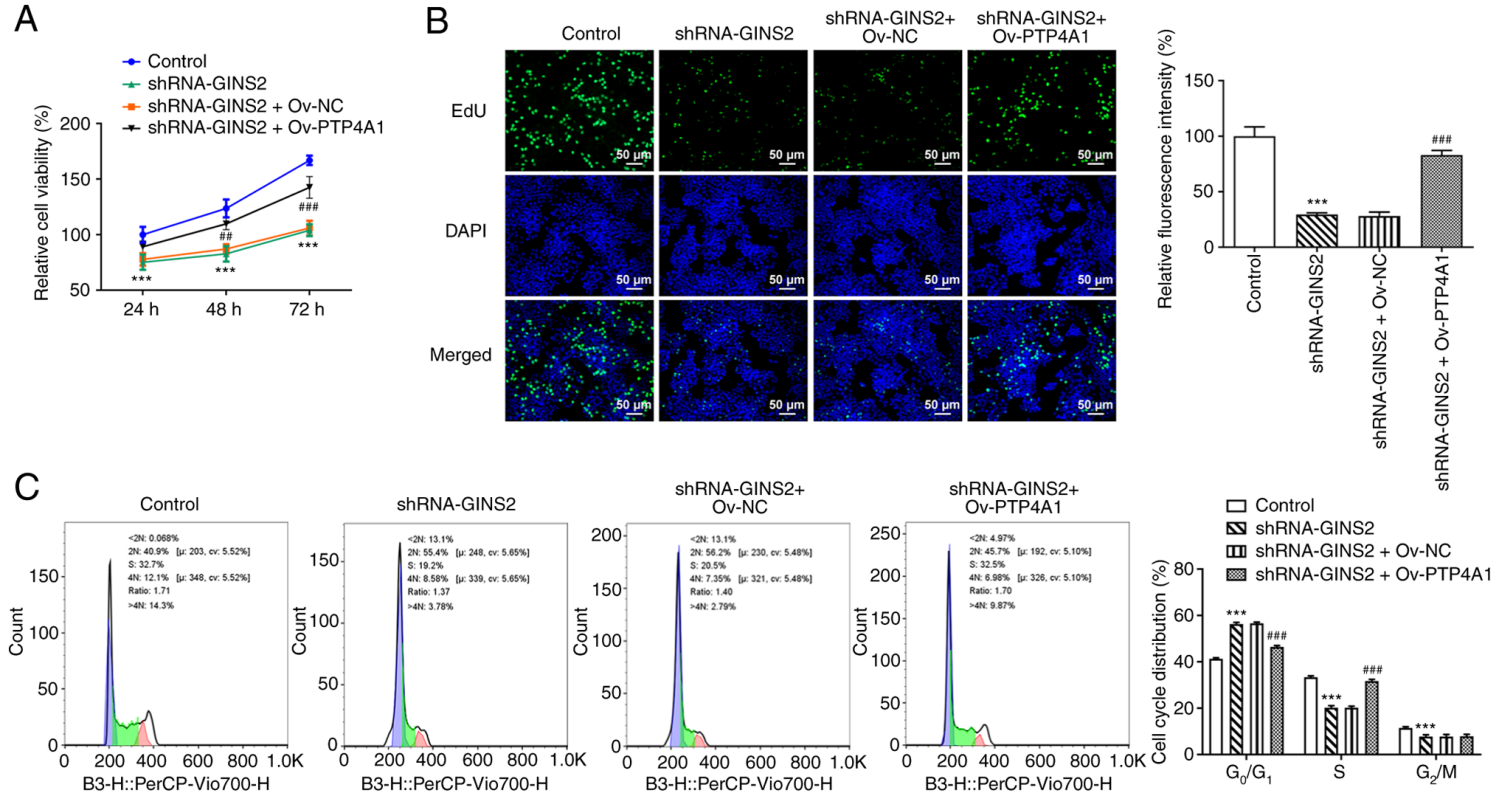

D
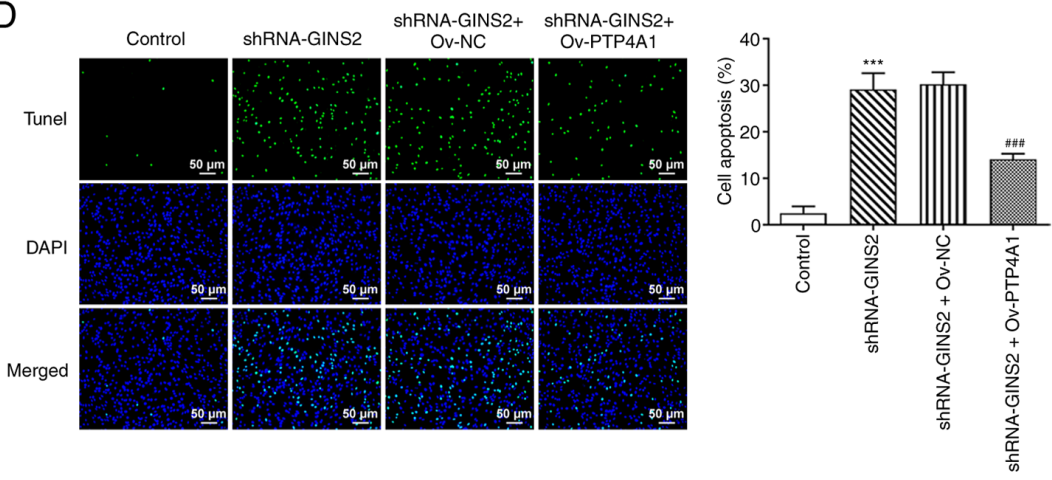

\section{E}
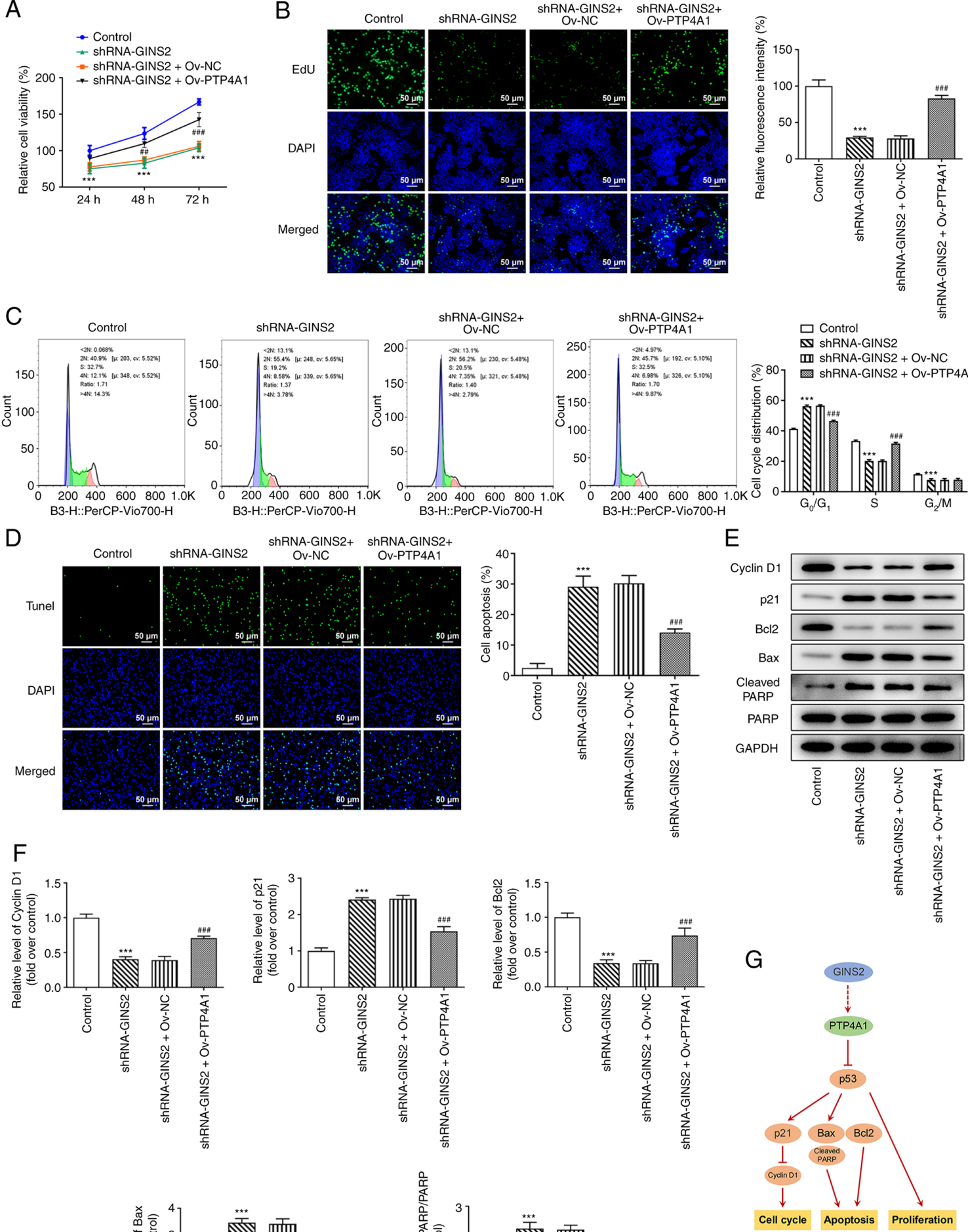
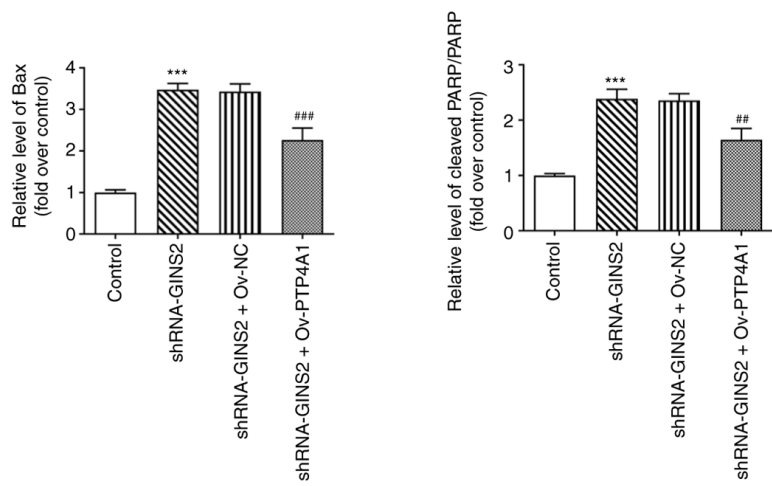


\section{Discussion}

Novel indicators for determining the prognosis of patients with colon cancer are required for the development of effective treatment options (1). GINS2 has been identified as a crucial regulator in cell cycle progression (23). It has also been reported that GINS2 promotes cell proliferation and apoptosis desensitization (24). The present results demonstrated that GINS2 knockdown inhibited the proliferation and induced cell cycle arrest and apoptosis in colon cancer. GINS2 may serve a key role in colon cancer.

The cell cycle is a complex process that provides the tumor cell with the opportunity to repair its damaged DNA (25). This process is regulated by numerous protein families, including cyclin-dependent kinases (CDKs), which drive cell cycle progression $(26,27)$. Cyclin D1, in association with CDK4/6, functions as an important regulator of the cell cycle (28). As a CDK inhibitor, p21 mainly serves a suppressive role in cell cycle progression (29). Apoptosis, also known as programmed cell death, is a physiological process that occurs in multicellular organisms (30). Chen et al (31) demonstrate that apoptosis acts as a critical participant in numerous biological processes, including tissue development and organ formation. Downregulation of GINS2 induces cell cycle arrest and apoptosis in lung cancer A549 cells (32). Silencing of GINS2, which is overexpressed in melanoma, inhibits cell proliferation and increases apoptosis in A375 cells (33). GINS2 interference induces cell cycle arrest and apoptosis of pancreatic cancer via the MAPK/ERK pathway (34). The results of the present study demonstrated that GINS2 knockdown induced cell cycle arrest and promoted apoptosis in colon cancer. In addition, the results of the present study also demonstrated that GINS2 knockdown reduced the expression levels of the anti-apoptotic protein $\mathrm{Bcl} 2$, but increased the expression levels of the pro-apoptotic proteins Bax and cleaved PARP.

PTP4A1 expression is increased in numerous cancers, including intrahepatic cholangiocarcinoma (18), non-small cell lung cancer (35) and cervical cancer (36). According to the Biogrid database, GINS2 has the ability to interact with PTP4A1, which was subsequently confirmed using a Co-IP assay. In addition, PTP4A1 expression levels were increased in colon cancer cells and the increased levels of PTP4A1 expression were decreased following transfection with the shRNA-GINS2 plasmids.

p53, a tumor suppressor, is described as a co-immunoprecipitating protein by Kress et al (37). Through the transcription regulation of downstream target genes involved in cell cycle arrest, apoptosis, DNA repair and metabolism, p53 exerts multiple biological functions in human diseases $(38,39)$. In addition, p53 influences the sensitivity of colon cancer cells to bleomycin (40). Mutant p53 is shown to promote angiogenesis in colon cancer, leading to a poor prognosis (41). As a regulatory target gene of p53, PTP4A1 activates p53 expression (21). The results of the present study demonstrated that GINS2 knockdown led to the activation of the p53 pathway through PTP4A1. Additionally, rescue experiments confirmed that the GINS2 knockdown-mediated suppression in cell proliferation and the increased levels of cell cycle arrest and apoptosis in colon cancer cells, were restored following PTP4A1 overexpression. These results highlighted that GINS2 knockdown exerted its regulatory effects on the proliferation, cycle arrest and apoptosis of colon cancer cells via regulation of the PTP4A1/p53 pathway. However, a key limitation of the present study was the lack of detection of GINS2 and PTP4A1 expression in clinical samples. Therefore, future studies should validate the results of the present study by performing some in-vivo experiments.

In conclusion, it was demonstrated that GINS2 regulated the proliferation, cell cycle progression and apoptosis of colon cancer cells through PTP4A1, indicating that GINS2 may serve as a biomarker for the development of novel therapies for colon cancer.

\section{Acknowledgements}

Not applicable.

\section{Funding}

No funding was received.

\section{Availability of data and materials}

The datasets used and/or analyzed during the current study are available from the corresponding author on reasonable request.

\section{Authors' contributions}

ZL designed the study and supervised the project. HH and LY performed the experiments and analyzed data. HH drafted the manuscript. All authors participated in the revision of the manuscript. ZL and $\mathrm{HH}$ confirm the authenticity of all the raw data. All authors read and approved the final manuscript.

\section{Ethics approval and consent to participate}

Not applicable.

\section{Patient consent for publication}

Not applicable.

\section{Competing interests}

The authors declare that they have no competing interests.

\section{References}

1. Chen H, Luo J and Guo J: Development and validation of a five-immune gene prognostic risk model in colon cancer. BMC Cancer 20: 395, 2020.

2. Bray F, Ferlay J, Soerjomataram I, Siegel RL, Torre LA and Jemal A: Global cancer statistics 2018: GLOBOCAN estimates of incidence and mortality worldwide for 36 cancers in 185 countries. CA Cancer J Clin 68: 394-424, 2018.

3. Siegel RL, Miller KD and Jemal A: Cancer Statistics, 2017. CA Cancer J Clin 67: 7-30, 2017.

4. Center MM, Jemal A, Smith RA and Ward E: Worldwide variations in colorectal cancer. CA Cancer J Clin 59: 366-378, 2009.

5. Edwards BK, Ward E, Kohler BA, Eheman C, Zauber AG, Anderson RN, Jemal A, Schymura MJ, Lansdorp-Vogelaar I, Seeff LC, et al: Annual report to the nation on the status of cancer, 1975-2006, featuring colorectal cancer trends and impact of interventions (risk factors, screening, and treatment) to reduce future rates. Cancer 116: 544-573, 2010 
6. Rupnarain C, Dlamini Z, Naicker S and Bhoola K: Colon cancer: Genomics and apoptotic events. Biol Chem 385: 449-464, 2004.

7. Cao C, Yan TD, Black D and Morris DL: A systematic review and meta-analysis of cytoreductive surgery with perioperative intraperitoneal chemotherapy for peritoneal carcinomatosis of colorectal origin. Ann Surg Oncol 16: 2152-2165, 2009.

8. Yaghoubi A, Khazaei M, Avan A, Hasanian SM and Soleimanpour S: The bacterial instrument as a promising therapy for colon cancer. Int J Colorectal Dis 35: 595-606, 2020.

9. Kubota Y, Takase Y, Komori Y, Hashimoto Y, Arata T, Kamimura $\mathrm{Y}$, Araki $\mathrm{H}$ and Takisawa $\mathrm{H}$ : A novel ring-like complex of Xenopus proteins essential for the initiation of DNA replication. Genes Dev 17: 1141-1152, 2003.

10. MacNeill SA: Structure and function of the GINS complex, a key component of the eukaryotic replisome. Biochem J 425: 489-500, 2010.

11. Stamova BS, Apperson M, Walker WL, Tian Y, Xu H, Adamczy P, Zhan X, Liu DZ, Ander BP, Liao IH, et al: Identification and validation of suitable endogenous reference genes for gene expression studies in human peripheral blood. BMC Med Genomics 2: 49, 2009.

12. Peng L, Song Z, Chen D, Linghu R, Wang Y, Zhang X, Kou X, Yang $J$ and Jiao S: GINS2 regulates matrix metallopeptidase 9 expression and cancer stem cell property in human triple negative Breast cancer. Biomed Pharmacother 84: 1568-1574, 2016.

13. Yan T, Liang W, Jiang E, Ye A, Wu Q and Xi M: GINS2 regulates cell proliferation and apoptosis in human epithelial ovarian cancer. Oncol Lett 16: 2591-2598, 2018.

14. Wei HB, Wen JZ, Wei B, Han XY and Zhang S: Expression and clinical significance of GINS complex in colorectal cancer Zhonghua Wei Chang Wai Ke Za Zhi 14: 443-447, 2011 (In Chinese).

15. Kabir MF, Mohd Ali J and Haji Hashim O: Microarray gene expression profiling in colorectal (HCT116) and hepatocellular (HepG2) carcinoma cell lines treated with Melicope ptelefolia leaf extract reveals transcriptome profiles exhibiting anticancer activity. PeerJ 6: e5203, 2018.

16. Achiwa $\mathrm{H}$ and Lazo JS: PRL-1 tyrosine phosphatase regulates c-Src levels, adherence, and invasion in human lung cancer cells. Cancer Res 67: 643-650, 2007.

17. Wang J, Kirby CE and Herbst R: The tyrosine phosphatase PRL-1 localizes to the endoplasmic reticulum and the mitotic spindle and is required for normal mitosis. J Biol Chem 277: 46659-46668, 2002

18. Liu LZ, He YZ, Dong PP, Ma LJ, Wang ZC, Liu XY, Duan M, Yang LX, Shi JY, Zhou J, et al: Protein tyrosine phosphatase PTP4A1 promotes proliferation and epithelial-mesenchymal transition in intrahepatic cholangiocarcinoma via the PI3K/AKT pathway. Oncotarget 7: 75210-75220, 2016.

19. Wang Y, Li ZF, He J, Li YL, Zhu GB, Zhang LH and Li YL: Expression of the human phosphatases of regenerating liver (PRLs) in colonic adenocarcinoma and its correlation with lymph node metastasis. Int J Colorectal Dis 22: 1179-1184, 2007.

20. Chi F, Wang Z, Li Y and Chang N: Knockdown of GINS2 inhibits proliferation and promotes apoptosis through the p53/GADD45A pathway in non-small-cell lung cancer. Biosci Rep 40: BSR20193949, 2020.

21. Min SH, Kim DM, Heo YS, Kim YI, Kim HM, Kim J, Han YM, Kim IC and Yoo OJ: New p53 target, phosphatase of regenerating liver 1 (PRL-1) downregulates p53. Oncogene 28: 545-554, 2009.

22. Livak KJ and Schmittgen TD: Analysis of relative gene expression data using real-time quantitative PCR and the 2(-Delta Delta C(T)) method. Methods 25: 402-408, 2001.
23. Kang YH, Galal WC, Farina A, Tappin I and Hurwitz J: Properties of the human Cdc45/Mcm2-7/GINS helicase complex and its action with DNA polymerase epsilon in rolling circle DNA synthesis. Proc Natl Acad Sci USA 109: 6042-6047, 2012.

24. Zhang X, Zhong L, Liu BZ, Gao YJ, Gao YM and Hu XX: Effect of GINS2 on proliferation and apoptosis in leukemic cell line. Int J Med Sci 10: 1795-1804, 2013.

25. Schwartz GK and Shah MA: Targeting the cell cycle: A new approach to cancer therapy. J Clin Oncol 23: 9408-9421, 2005.

26. Swanton C: Cell-cycle targeted therapies. Lancet Oncol 5: 27-36, 2004.

27. Hydbring P, Malumbres M and Sicinski P: Non-canonical functions of cell cycle cyclins and cyclin-dependent kinases. Nat Rev Mol Cell Biol 17: 280-292, 2016.

28. Tchakarska G and Sola B: The double dealing of cyclin D1. Cell Cycle 19: 163-178, 2020.

29. Karimian A, Ahmadi Y and Yousefi B: Multiple functions of p21 in cell cycle, apoptosis and transcriptional regulation after DNA damage. DNA Repair (Amst) 42: 63-71, 2016.

30. Kerr JF: History of the events leading to the formulation of the apoptosis concept. Toxicology 181-182: 471-474, 2002.

31. Chen M, Wu W, Liu D, Lv Y, Deng H, Gao S, Gu Y, Huang M, Guo X, Liu B, et al: Evolution and Structure of API5 and Its Roles in Anti-Apoptosis. Protein Pept Lett 28: 612-622, 2021.

32. Sun D, Zong Y, Cheng J, Li Z, Xing L and Yu J: GINS2 attenuates the development of lung cancer by inhibiting the STAT signaling pathway. J Cancer 12: 99-110, 2021.

33. Hao YQ, Liu KW, Zhang X, Kang SX, Zhang K, Han W, Li L and Li ZH: GINS2 was regulated by lncRNA XIST/miR-23a-3p to mediate proliferation and apoptosis in A375 cells. Mol Cell Biochem 476: 1455-1465, 2021.

34. Zhang M, He S, Ma X, Ye Y, Wang G, Zhuang J, Song Y and Xia W: GINS2 affects cell viability, cell apoptosis, and cell cycle progression of pancreatic cancer cells via MAPK/ERK pathway. J Cancer 11: 4662-4670, 2020.

35. Wang T, Shi X, Wang Z, Liu X, Zhang G, Zhu Q, Mi L and Wang R: Overexpression of PTP4A1 is associated with poor overall survival in non-small cell lung cancer. Int J Clin Exp Pathol 11: 3583-3590, 2018

36. Li X, Ma N, Zhang Y, Wei H, Zhang H, Pang X, Li X, Wu D, Wang D, Yang Z and Zhang S: Circular RNA circNRIP1 promotes migration and invasion in cervical cancer by sponging miR-629-3p and regulating the PTP4A1/ERK1/2 pathway. Cell Death Dis 11: 399, 2020

37. Kress M, May E, Cassingena R and May P: Simian virus 40-transformed cells express new species of proteins precipitable by anti-simian virus 40 tumor serum. J Virol 31: 472-483, 1979.

38. Lane D and Levine A: p53 Research: The past thirty years and the next thirty years. Cold Spring Harb Perspect Biol 2: a000893, 2010.

39. Levav-Cohen Y, Goldberg Z, Tan KH, Alsheich-Bartok O, Zuckerman V, Haupt S and Haupt Y: The p53-Mdm2 loop: A critical juncture of stress response. Subcell Biochem 85: 161-186, 2014.

40. Lee YS, Yoon S, Park MS, Kim JH, Lee JH and Song CW: Influence of $\mathrm{p} 53$ expression on sensitivity of cancer cells to bleomycin. J Biochem Mol Toxicol 24: 260-269, 2010.

41. Takahashi Y, Bucana CD, Cleary KR and Ellis LM: p53, vessel count, and vascular endothelial growth factor expression in human colon cancer. Int J Cancer 79: 34-38, 1998.

This work is licensed under a Creative Commons Attribution-NonCommercial-NoDerivatives 4.0 International (CC BY-NC-ND 4.0) License. 